\title{
RAYLEIGH-WAVE COUPLING TO ATMOSPHERIC COMPRESSIONN WAVES*
}

\author{
By W. S. Jardetzky and Frank Press
}

\begin{abstract}
The THEory of dispersive Rayleigh waves coupled to atmospheric compressional waves is derived for the case of a solid surface layer. Numerical computation of phase and group velocity curves indicates that an additional branch may be introduced to the dispersion curves as a result of air coupling. Amplitudes of waves propagated according to the various branches are briefly discussed.
\end{abstract}

\section{INTRODUCTION}

RECENT experimental and theoretical work has indicated that coupling of surface waves to the atmosphere is not an uncommon phenomenon. Press, Crary, Oliver and Katz $z^{1}$ identified air-coupled flexural waves on a floating ice sheet. Press and Ewing $^{2}$ made use of Lamb's elementary theory ${ }^{3}$ to show that coupling between air waves and surface waves of all types is appreciable when the phase velocity of the surface wave is very close to the speed of sound in air. These authors also developed the exact theory for air-coupled flexural waves in a floating ice sheet and presented numerical computation of dispersion and relative amplitudes.

An experimental investigation of air coupling to Rayleigh waves has recently been completed with the coöperation of the Field Research Laboratory of Magnolia Petroleum Company. ${ }^{4}$ Coupling of the atmosphere with hydrodynamic gravity waves is now being investigated.

In this paper, the theory for air-coupled Rayleigh waves originating in an impulsive point source in the air, is outlined for the case of a solid surface layer. Numerical computation of dispersion is made for a particular case, the results being applicable for a source in the air or in the ground.

\section{THEORY}

The existence of air-coupled Rayleigh waves was shown by Bateman ${ }^{5}$ on the assumpof a homogeneous earth. Lee investigated the propagation of Rayleigh waves in two solid layers. ${ }^{6}$

We now consider Rayleigh Waves coupled to atmospheric compressional waves for the case of a three-layered space formed by air in the half space $z=0$ to $z=-\infty$, an "upper" solid layer (1) between the planes $z=0$ and $z=H$ and a "lower" layer (2) between $z=H$ and $z=\infty$. We assume a point source in the air at a distance $d$

* The research reported in this paper has been made possible through support and sponsorship by the Geophysical Research Division of the Cambridge Air Force Research Center. Manuscript received for publication March 9, 1951.

${ }^{1}$ Frank Press, A. P. Crary, Jack Oliver, and Sam Katz, "Air-coupled Flexural Waves in Floating Ice," Trans. Am. Geophys. Union, 32:166-172 (1951).

${ }_{2}$ Frank Press and Maurice Ewing, "Theory of Air-coupled Flexural Waves," Jour. Appl. Physics, 22:892-899 (1951).

${ }^{3}$ H. Lamb, "On Waves Due to a Traveling Disturbance, with an Application to Waves in Superposed Fluids," Phrl. Mag., $31: 387$ (1951).

${ }^{4}$ Frank Press and Maurice Ewing, "Ground-Roll Coupling to Atmospheric Compressional Waves," Geophysics, 16:416-430 (1951).

5 H. Bateman, "Rayleigh Waves," Proc. Nat. Acad. Sci., 24:315-320 (1938).

"A. W. Lee, "The Effect of Geological Structure upon Microseismic Disturbance," Mon. Not. Roy. Astron. Soc., Geophys. Suppl., 3:83-105 (1932); idem, "Further Investigation of the Effect of Geologic Structure on Microseismic Disturbance," ibid., 3:238-252 (1934). 
above the plane $z=0$. We denote by $\rho_{0}, \rho_{1}, \rho_{2}, \alpha_{0}, \alpha_{1}, \alpha_{2}, \beta_{1}, \beta_{2}$ the densities and velocities of dilatational and shear waves in the air and the elastic media 1 and 2.

Applying the well-known method of Lamb, ${ }^{7}$ we represent the horizontal component of displacement, $q$, and the vertical component, $w$, by the formulas

$$
\begin{aligned}
& q=\frac{\partial \phi}{\partial r}+\frac{\partial^{2} \psi}{\partial r \partial z} \\
& w=\frac{\partial \phi}{\partial z}+\frac{\partial^{2} \psi}{\partial z^{2}}+\frac{\omega^{2}}{\beta^{2}} \psi
\end{aligned}
$$

where $r$ and $z$ are two cylindrical coördinates. Simple harmonic motion of the form $e^{i w t}$ has been assumed. The differential equations of motion may be transformed to the following system:

$$
\begin{array}{ll}
\left(\nabla^{2}+\frac{\omega^{2}}{\alpha_{0}^{2}}\right) \phi_{0}=0, & \left(\nabla^{2}+\frac{\omega^{2}}{\beta_{1}^{2}}\right) \psi_{1}=0, \\
\left(\nabla^{2}+\frac{\omega^{2}}{\alpha_{1}^{2}}\right) \phi_{1}=0, & \left(\nabla^{2}+\frac{\omega^{2}}{\beta_{2}^{2}}\right) \psi_{2}=0 .
\end{array}
$$

In order to find the functions $\phi_{i}$ and $\psi_{i}$ which satisfy equations (2), we must specify the boundary conditions. The stresses

$$
\begin{aligned}
& p_{z z}=\lambda \Delta+2 \mu \frac{\partial w}{\partial z}=\lambda \nabla^{2} \phi+2 \mu \frac{\partial w}{\partial z} \\
& p_{z r}=\mu\left(\frac{\partial q}{\partial z}+\frac{\partial w}{\partial r}\right)
\end{aligned}
$$

where $\lambda$ and $\mu$ are Lamé's constants, satisfy the conditions

$$
\left(p_{z z}\right)_{1}=\left(p_{z z}\right)_{0},\left(p_{z r}\right)_{1}=0 \quad \text { at } z=0
$$

and

$$
\left(p_{z z}\right)_{2}=\left(p_{z z}\right)_{1}, \quad\left(p_{z r}\right)_{2}=\left(p_{z r}\right)_{1} \quad \text { at } \quad z=H .
$$

If we assume that no voids are formed at the interface between the air and the upper layer, we may write

$$
w_{1}=w_{0} \quad \text { at } \quad z=0
$$

and, if a welded connection between the two solid layers is assumed, we have

$$
w_{2}=w_{1}, \quad q_{2}=q_{1} \quad \text { at } z=H .
$$

${ }^{7}$ H. Lamb, "On the Propagation of Tremors over the Surface of an Elastic Solid," Phil. Trans. Roy. Soc. London, Ser. A, 203:1-42, (1904). 
It is also assumed that the displacements vanish at $z= \pm \infty$. Following Lamb, ${ }^{8}$ we introduce two conditions which must be satisfied at the horizontal plane passing through the source $(z=d)$, namely, the pressure must be continuous

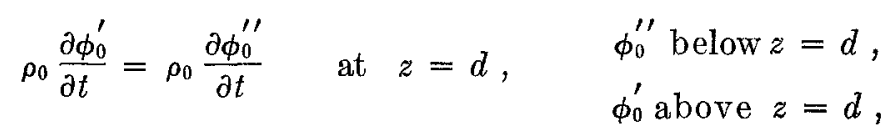

and the vertical component of the velocity is discontinuous at the plane $z=d$

$$
\frac{\partial \phi_{0}^{\prime}}{\partial z}-\frac{\partial \phi_{0}^{\prime \prime}}{\partial z}=2 Y J(k r)
$$

The fundamental solutions of equations (2) satisfying all above conditions must be written in the well-known form

$$
\begin{array}{rrr}
\phi_{0}^{\prime}=A \exp \left(\gamma_{0} z\right) J_{0}(k r), & |z|>|d| \\
\phi_{0}^{\prime \prime}=\left\{B \exp \left(-\gamma_{0} z\right)+C \exp \left(\gamma_{0} z\right)\right\} & J_{0}(k r),|d| \geqq|z| \geqq 0 \\
\phi_{1}=\left\{D \exp \left(\gamma_{1} z\right)+E \exp \left(-\gamma_{1} z\right)\right\} & J_{0}(k r), & 0 \leqq z \leqq H \\
\phi_{2}=F \exp \left(-\gamma_{2} z\right) J_{0}(k r), & H<z<\infty \\
\psi_{1}=\left\{M \exp \left(\gamma_{3} z\right)+N \exp \left(-\gamma_{3} z\right)\right\} & J_{0}(k r), & 0 \leqq z \leqq H \\
\psi_{2}=P \exp \left(-\gamma_{4} z\right) J_{0}(k r), & H<z<\infty .
\end{array}
$$

where $J_{0}(k r)$ is the Bessel function and the positive real or positive imaginary constants $\gamma_{i}$ are given by relations

$$
\begin{aligned}
& \gamma_{0}^{2}=k^{2}-\frac{\omega^{2}}{\alpha_{0}^{2}}, \quad \gamma_{1}^{2}=k^{2}-\frac{\omega^{2}}{\alpha_{1}^{2}}, \quad \gamma_{2}^{2}=k^{2}-\frac{\omega^{2}}{\alpha_{2}^{2}}, \\
& \gamma_{3}^{2}=k^{2}-\frac{\omega^{2}}{\beta_{1}^{2}}, \quad \gamma_{4}^{2}=k^{2}-\frac{\omega^{2}}{\beta_{2}^{2}} .
\end{aligned}
$$

in order to satisfy equations (2). Nine arbitrary constants, $A, B, C, \ldots P$ figuring in the expressions (11)-(16) may be chosen so that the nine conditions (5), (6), (7), (8), (9), and (10) are satisfied.

On substituting the functions (11)-(16) in these boundary conditions, we obtain the following system of equations to determine the values of $A, B, C, \ldots P$ :

$$
\begin{gathered}
\omega^{2} \rho_{0}(B+C)+\left(2 \mu_{1} k^{2}-\rho_{1} \omega^{2}\right)(D+E)+2 \mu_{1} \gamma_{3} k^{2}(M-N)=0 \\
2 \gamma_{1}(D-E)+\left(\gamma_{3}^{2}+k^{2}\right)(M+N)=0 \\
\left(2 \mu_{1} k^{2}-\rho_{1} \omega^{2}\right)\left(D e^{\gamma_{1} H}+E e^{-\gamma_{1} H}\right)-\left(2 \mu_{2} k^{2}-\rho_{2} \omega^{2}\right) F e^{-\gamma_{2} H} \\
+2 \mu_{1} \gamma_{3} k^{2}\left(M e^{\gamma_{3} H}-N e^{-\gamma_{3} H}\right)+2 \mu_{2} \gamma_{4} k^{2} P e^{-\gamma_{4} H}=0 \\
\frac{2 \mu_{1} \gamma_{1}\left(D e^{\gamma_{1} H}-E e^{-\gamma_{1} H}\right)+2 \mu_{2} \gamma_{2} F e^{-\gamma_{2} H}+\mu_{1}\left(\gamma_{3}^{2}+k^{2}\right)\left(M e^{\gamma_{3} H}+N e^{-\gamma_{3} H}\right)}{{ }^{8} \text { Ibid. }}
\end{gathered}
$$




$$
\begin{gathered}
-\mu_{2}\left(\gamma_{4}^{2}+k^{2}\right) P e^{-\gamma_{4} H}=0 \\
\gamma_{0}(-B+C)-\gamma_{1}(D-E)-k^{2}(M+N)=0 \\
\gamma_{1}\left(D e^{\gamma_{1} H}-E e^{-\gamma_{1} H}\right)+\gamma_{2} F e^{-\gamma_{2} H}+k^{2}\left(M e^{\gamma_{3} H}+N e^{-\gamma_{3} H}\right)-k^{2} P e^{-\gamma_{4} H}=0 \\
D e^{\gamma_{1} H}+E e^{-\gamma_{1} H}-F e^{-\gamma_{2} H}+\gamma_{3}\left(M e^{\gamma_{3} H}-N e^{-\gamma_{3} H}\right)+\gamma_{4} P e^{-\gamma_{4} H}=0 \\
A e^{\gamma_{0} d}-B e^{-\gamma_{0} d}-C e^{\gamma_{0} d}=0 \\
\gamma_{0} A e^{\gamma_{0}^{d}}+\gamma_{0}\left(B e^{-\gamma_{0} d}-C e^{\gamma_{0} d}\right)=2 Y .
\end{gathered}
$$

Two of the coefficients $A, B, \ldots P$ may be easily eliminated. From the two last equations it follows that

and

$$
B=\frac{Y}{\gamma_{0}} e^{\gamma_{0} d}
$$

$$
C=A-\frac{Y}{\gamma_{0}} e^{-\gamma_{0^{t}}} .
$$

Therefore, the equations (18)-(24) take the form

$$
\begin{aligned}
a_{1} A+ & d_{1} D+e_{1} E+m_{1} M+n_{1} N=-2 \rho_{0} \omega^{2} \frac{Y}{\gamma_{0}} \sinh \left(\gamma_{0} d\right) \\
& d_{2} D+e_{2} E+m_{2} M+n_{2} N=0 \\
& d_{3} D+e_{3} E+m_{3} M+n_{3} N+f_{3} F+p_{3} P=0 \\
& d_{4} D+e_{4} E+m_{4} M+n_{4} N+f_{4} F+p_{4} P=0 \\
a_{5} A+ & d_{5} D+e_{5} E+m_{5} M+n_{5} N=-2 Y \cosh \left(\gamma_{0} d\right) \\
& d_{6} D+e_{6} E+m_{6} M+n_{6} N+f_{6} F+p_{6} P=0 \\
& d_{7} D+e_{7} E+m_{7} M+n_{7} N+f_{7} F+p_{7} P=0
\end{aligned}
$$

Since the determinant of these seven equations is not identically equal to zero, we can find definite values of the coefficients $A, B, \ldots P$. We can then apply the method of $\mathrm{Lamb}^{9}$ and generalize the discontinuity of vertical velocity using the Fourier-Bessel integral by taking $Y=k d k$ and integrating with respect to $k$ from 0 to $\infty$. Then we can obtain solutions satisfying all conditions, including the requirements for a point source. It may be difficult to evaluate the integrals representing the solutions, since the quantities $A, B, \ldots P$ are complicated, owing to the high order of the determinant.

We restrict ourselves at this point to the analysis of the frequency equation which is given by the condition that the foregoing determinant is equal to zero.

Since the coefficients $a, d, \ldots$ of the system (29) depend on the elastic constants and on the quantities $\rho_{i}, \alpha_{i}, \beta_{i}$, and $\gamma_{i}$, which may be expressed as functions of the phase velocity, $c=\omega / k$, this determinant may be considered as a function of the phase velocity $c$ and of the frequency $f=k c / 2 \pi$. Equating the determinant to zero, we therefore obtain an equation which defines an implicit relationship between frequency and the phase velocity.

${ }^{9}$ Ibid. 
Rewriting the frequency equation, we omit all factors which cannot be equal to zero and transform the remaining determinant to reduce the number of elements. After these transformations, the frequency equation takes the form:
$1 \frac{2 \mu_{1} k^{2}-\rho_{1} \omega^{2}}{\rho_{0} \omega^{2} \cosh \left(\gamma_{1} H\right)}$
$0 \quad \frac{2 \mu_{1} \gamma_{3} k^{2}}{\rho_{0} \omega^{2} \sinh \left(\gamma_{3} H\right)}$
0
0
0
$\frac{2 \gamma_{1}}{\sinh \left(\gamma_{1} H\right)} \frac{2 k^{2} \beta_{1}^{2}-\omega^{2}}{\beta_{1}^{2} \cosh \left(\gamma_{3} H\right)}$
0
0
$0-\rho_{1} \omega^{2} \quad-\rho_{1} \omega^{2}$
0
$0 \quad \rho_{2} \omega^{2}+2\left(\mu_{1}-\mu_{2}\right) k^{2} 2\left(\mu_{2}-\mu_{1}\right) \gamma_{4} k^{2}$
0
0
0
$-\rho_{1} \omega^{2}$
$-\rho_{1} \omega^{2}$
$2\left(\mu_{2}-\mu_{1}\right) \gamma_{2} \quad 2\left(\mu_{1}-\mu_{2}\right) k^{2}$
$+\rho_{2} \omega^{2}$
$0-\frac{2 \mu_{1} k^{2}-\rho_{1} \omega^{2}}{\rho_{0} \omega^{2} \cosh \left(\gamma_{1} H\right)}$
$0 \frac{-\omega^{2}}{2 \gamma_{0} \cosh \left(\gamma_{3} H\right) \beta_{1}{ }^{2}} \frac{-2 \mu_{1} \gamma_{3} k^{2}}{\rho_{0} \omega^{2} \sinh \left(\gamma_{3} H\right)}$
0
0
$0 \gamma_{1} \tanh \left(\gamma_{1} H\right) \quad \gamma_{1} \operatorname{cotanh}\left(\gamma_{1} H\right)$
$k^{2}$
$k^{2} \quad \gamma_{2}$
0
$1 \quad \begin{array}{llll}\gamma_{3} \tanh \left(\gamma_{3} H\right) & \gamma_{3} \operatorname{cotanh}\left(\gamma_{3} H\right) & -1\end{array}$
$\gamma_{4}$

Developing the determinant, we put

and

$$
V=\frac{c}{\beta_{1}}
$$

$$
\begin{aligned}
n_{0}=\sqrt{1-V^{2} \frac{\beta_{1}^{2}}{\alpha_{0}^{2}},} \quad n_{1}=\sqrt{1-V^{\tau} \frac{\beta_{1}^{2}}{\alpha_{1}^{2}}}, \quad n_{2}=\sqrt{1-V^{2} \frac{\beta_{1}^{2}}{\alpha_{2}^{2}}} \\
n_{3}=\sqrt{1-V^{2}}, \quad n_{4}=\sqrt{1-V^{2} \frac{\beta_{1}^{2}}{\beta_{2}^{2}}}
\end{aligned}
$$

Introducing Lee's notations ${ }^{10}$

$$
\begin{aligned}
& X=\frac{\rho_{2}}{\rho_{1}} V^{2}-2\left(\frac{\mu_{2}}{\mu_{1}}-1\right) \\
& Y=V^{2}+2\left(\frac{\mu_{2}}{\mu_{1}}-1\right)=V^{2}+W \\
& Z=\frac{\rho_{2}}{\rho_{1}} V^{2}-V^{2}-2\left(\frac{\mu_{2}}{\mu_{1}}-1\right)=X-V^{2} \\
& W=2\left(\frac{\mu_{2}}{\mu_{1}}-1\right),
\end{aligned}
$$

${ }^{10}$ A. W. Lee, opp. citt (see fn. 6, above). 
we put

$$
G_{1}=X Y-n_{2} n_{4} W Y, \quad G_{2}=Z^{2}-n_{2} n_{4} Y^{2}, \quad G_{3}=n_{2} n_{4} W^{2}-X^{2},
$$

and

$$
\begin{aligned}
& l_{0}=4\left(2-V^{2}\right) G_{1} \\
& l_{1}=\left(2-V^{2}\right)^{2} \cdot \frac{1}{n_{1} n_{3}} G_{2}-4 n_{1} n_{3} G_{3}-\frac{\rho_{0} \rho_{2}}{\rho_{1}^{2}} \frac{n_{1} n_{4}}{n_{0} n_{3}} V^{8} \\
& l_{2}=-\left(2-V^{2}\right)^{2} \frac{\rho_{2}}{\rho_{1}} \frac{n_{2}}{n_{1}} V^{4}+\frac{4 \rho_{2}}{\rho_{1}} n_{1} n_{4} V^{2}+\frac{\rho_{0}}{\rho_{1}} \frac{n_{1}}{n_{0}} V^{4} G_{3} \\
& l_{3}=-\left(2-V^{2}\right)^{2} \frac{\rho_{2}}{\rho_{1}} \frac{n_{4}}{n_{3}} V^{4}+\frac{4 \rho_{2}}{\rho_{1}} n_{2} n_{3} V^{4}+\frac{\rho_{0}}{\rho_{1}} \cdot \frac{1}{n_{0} n_{3}} V^{4} G_{2} \\
& l_{4}=\left(2-V^{2}\right)^{2} G_{3}-4 G_{2}-\frac{\rho_{0} \rho_{2}}{\rho_{1}^{2}} \frac{n_{2}}{n_{0}} V^{8} .
\end{aligned}
$$

The frequency equation then takes the form

$$
\begin{aligned}
& l_{0}+l_{1} \sinh \left(n_{1} k H\right) \sinh \left(n_{3} k H\right)+l_{2} \sinh \left(n_{1} k H\right) \cosh \left(n_{3} k H\right) \\
& \quad+l_{3} \cosh \left(n_{1} k H\right) \sinh \left(n_{3} k H\right)+l_{4} \cosh \left(n_{1} k H\right) \cosh \left(n_{3} k H\right)=0 .
\end{aligned}
$$

The last terms of the coefficients $l_{i}$ having the factors $\rho_{0}$ and $1 / n_{0}$ represent the influence of the air. For numerical computations, it was assumed that Poisson's ratio $\sigma=0.25$ for both solid layers, i.e., $\lambda_{1}=\mu_{1}$ and $\lambda_{2}=\mu_{2}$. The following data were used:

$$
\begin{gathered}
\alpha_{0}=1070 \mathrm{ft} / \mathrm{sec} ., \beta_{1}=800 \mathrm{ft} / \text { sec. } \\
\frac{\rho_{2}}{\rho_{1}}=1.39, \quad \frac{\rho_{0}}{\rho_{1}}=0.001, \quad \frac{\mu_{2}}{\mu_{1}}=13.77 .
\end{gathered}
$$

These values were chosen as most representative of near-surface conditions often encountered in seismic prospecting. It is obvious that air-coupling effects are negligible when $0.9194 \beta_{1}>\alpha_{0}$, since $c>\alpha_{0}$ for this case, and the air-coupling term of the frequency equation which contains the factor $\rho_{0} / \rho_{1} n_{0}$ is very small.

In the case of air-coupled Rayleigh waves, real values of $k H$ must correspond to the interval

$$
0.9194 \leqq V \leqq \frac{\alpha_{0}}{\beta_{1}}
$$

The upper limit is equal, in this case, to $1.3375(=1070 / 800)$. For larger values of $V$, the roots of the frequency equation become complex. We have computed the real and imaginary parts of these roots. They are given in table 2 . All real roots computed, and the real parts of complex roots, are given in table 1 . The roots were computed by successive approximations, using Lee's computations to obtain the first approximation. Group velocity $U$ was obtained by graphical differentiation of the phase velocity curve, using the familiar equation

$$
U=c+k \frac{d c}{d k}
$$


TABLE 1

\begin{tabular}{c|c|c}
\hline$V=c / \beta_{1}$ & Lee's $k H$ & $k H$ \\
\hline 0.952 & 5.15 & 4.75 \\
.992 & 365 & 3.84 \\
110 & 2.86 & 2.88 \\
1.28 & 2.27 & 2.20 \\
1.30 & 2.21 & 2.15 \\
1.32 & 2.14 & 2.10 \\
1336 & 2.10 & 2.05 \\
1.337 & 2.10 & 2.02 \\
1.3374 & 2.09 & 1.98 \\
1.3375 & & 0 \\
1.3375 & & 45 \\
1.3376 & 2.09 & $207^{\mathrm{a}}$ \\
1.34 & 2.06 & $2.07^{\mathrm{a}}$ \\
1.38 & 1.98 & $1.98^{\mathrm{a}}$ \\
1.40 & 1.93 & $1.94^{\mathrm{a}}$ \\
1.41 & 1.90 & \\
1.65 & 153 & \\
1.98 & 1.12 & \\
2.15 & 0938 & \\
2.30 & 0806 & \\
2.48 & 0.648 & \\
2.53 & 0.610 & \\
2.64 & 0.462 & \\
2.73 & 0.294 & \\
2.81 & 0.143 & \\
2.89 & 0 & \\
\hline
\end{tabular}

a Real part.

TABLE 2

\begin{tabular}{c|c}
\hline \multicolumn{1}{c|}{} & $k H$ \\
\hline 1.3375 & 4.25 \\
1.3376 & $2.07+.08 i$ \\
134 & $2.07+.02 i$ \\
1.38 & $1.98+.01 i$ \\
1.40 & $194+.00 i$ \\
\hline
\end{tabular}

The phase velocity curve in the case of air-coupled Rayleigh waves differs very little from the curve given by Lee until the neighborhood of the critical point $V=1.3375$ is reached (fig. 1). At this point it deviates to the left and intersects the $V$-axis at a point

$$
1.3374<V<1.3375
$$

The real part of complex roots above the critical value is very close to the corresponding roots of the frequency equation without air-connected terms; but it deviates to the right in the neighborhood of the critical value, and "ends" at the point $k H=4.25$ of the line $V=1.3375$. 


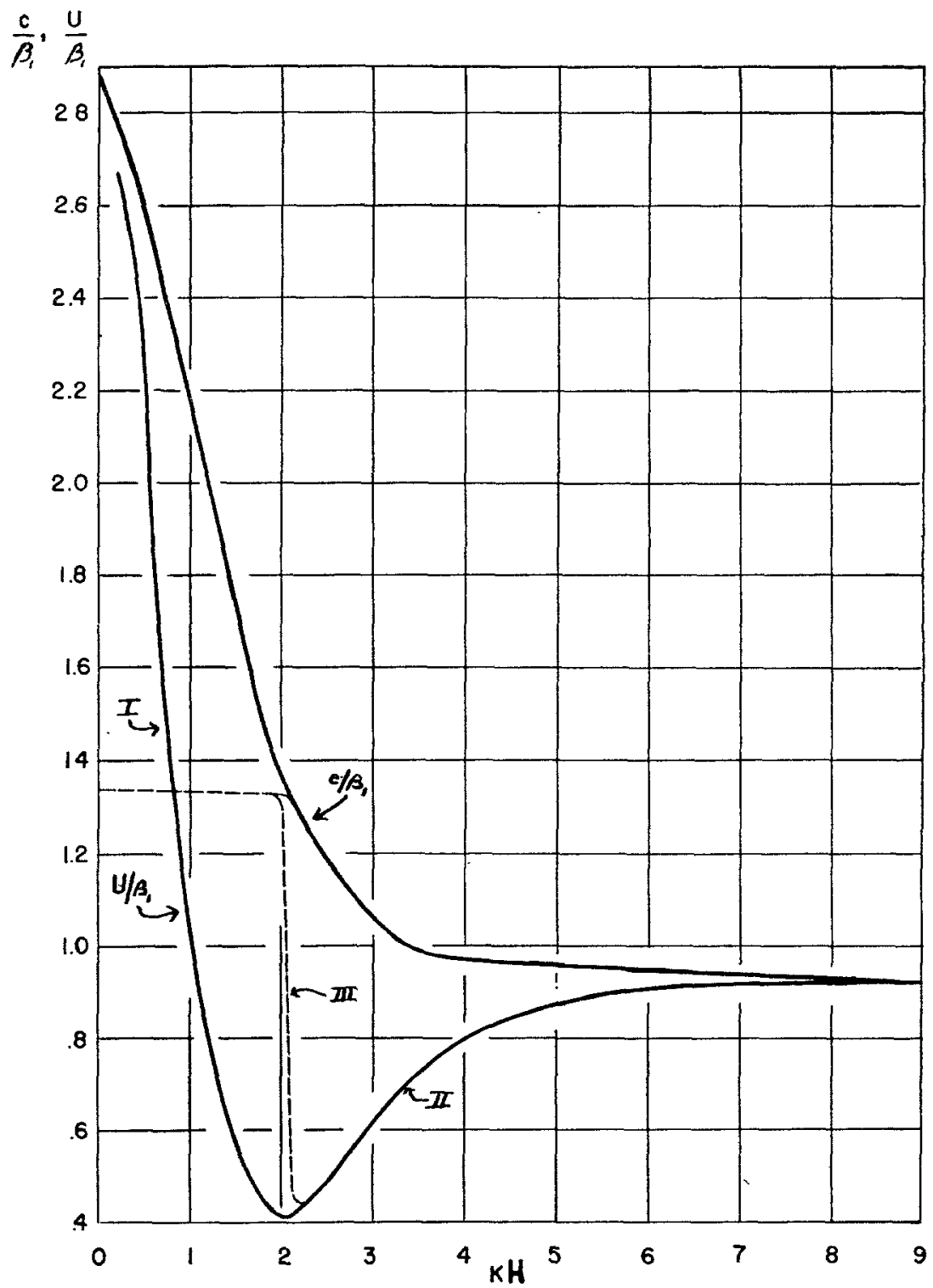

Fig. 1. Phase and group velocity curves for air-coupled Rayleigh waves.

Results of these computations are presented in dimensionless form in figure 1, where $c / \beta_{1}$ and $U / \beta_{1}$ are plotted against $k H$. The heavy lines indicate where atmospheric influence is negligible and the results are similar to those obtained by Lee. The dashed lines represent new branches introduced by air coupling.

It is well-known that the velocities and periods of waves propagated through a dispersive medium can be obtained from the corresponding group velocity curve. To obtain the amplitudes of waves corresponding to the different branches of the group velocity curve, one must compute excitation functions which depend upon 
the nature and position of the source, the location of the receivers, and implicitly on the roots of the frequency equation. Although the mathematical procedure is straightforward, the numerical work required is extremely lengthy. We can, therefore, discuss the frequencies and velocities of Rayleigh waves coupled to the atmosphere, but the relative amplitudes of waves for different source and receiver locations cannot be given. Existing calculations for air coupling to another type of surface wave can be used, however, to infer something about relative amplitudes.

The group velocity curve in figure 1 is divided into branches I, II, III, each of which represents a different train of waves. Branch I corresponds to the dispersive train of Rayleigh waves observed on seismograms of earthquakes. Branch I also accounts for the dispersive Rayleigh waves usually associated with ground roll. These waves first appear as long-period arrivals traveling with the speed of Rayleigh waves in the bottom layer, $U=0.9194 \beta_{2}$. Succeeding waves gradually decrease in period, since $k H$ increases as the group velocity decreases. Waves continue to arrive with decreasing period until a time corresponding to propagation at the minimum value of group velocity. From the analogous theory referred to above, we would expect waves propagated according to branch $\mathrm{I}$ to predominate for a source located within the solid layers recorded by a pickup on the solid layer. Waves with group velocity values near the minimum in branch I have phase velocities approaching the speed of sound in air from the side $c>\alpha_{\theta}$. These waves are attenuated since $k H$ is complex and has an increasingly large imaginary component as $c \rightarrow \alpha_{0}$ (table 2).

No significance should be attached to the occurrence of a minimum value of group velocity near the critical point $c=V_{0}$. Had we chosen other values for the elastic constants of the layers, the phase velocity corresponding to the minimal group velocity could have been much different from $V_{0}$.

Branch Ir represents a dispersive train of waves beginning as a high-frequency arrival at a time corresponding to propagation with the velocity of Rayleigh waves in the surface layer. The frequency of these waves decreases as time progresses until the time corresponding to propagation at the minimum group velocity, when the waves of branch I and branch Ir merge to form a single train of waves having a discrete frequency.

Branch III represents an additional train introduced by coupling of Rayleigh waves to atmospheric compressional waves. This train begins at a time corresponding to propagation at the speed of sound in air, and continues with almost constant frequency until the time $t=r / 0.44 \beta_{1}$. The phase velocity of these waves should be close to the speed of sound in air. From experimental and theoretical results referred to earlier, we might expect these waves to be prominent for a source in the air recorded by a pickup on the ground, and for a source within the ground recorded by a microphone in the air. There is an additional branch with dispersion features similar to branch III, corresponding to the complex phase velocities having real parts greater than, but close to, $\alpha_{0}$.

Higher modes of propagation exist at correspondingly higher frequencies. 


\section{CONCLUSIONS}

The existence of a surface layer in which the speed of Rayleigh waves is less than the speed of sound in air introduces a new branch to the dispersion curves, corresponding to a train of approximately constant frequency waves beginning with the arrival of the air wave and continuing to a time depending on the distance and the elastic constants of the layers. It is probable that these new waves are especially prominent for a source within the air recorded with a seismograph and for a source within the ground recorded with a microphone.

Lamont Geological Observatory

(ColdMbia University)

Contribution No. 54 . 